\title{
Electronic and magnetic structures of $\mathrm{Sr}_{2} \mathrm{FeMoO}_{6}$
}

\author{
Sugata Ray, ${ }^{\star}$ Ashwani Kumar, ${ }^{\star}$ D. D. Sarma, ${ }^{\star} \otimes$ R. Cimino, ${ }^{\S}$ S. Turchini,, S. Zennaro ${ }^{\llbracket}$ and N. Zema ${ }^{\sharp}$ \\ ${ }^{\star}$ Solid State and Structural Chemistry Unit, Indian Institute of Science, Bangalore 560 012, INDIA \\ ${ }^{\S}$ Istituto Nazionale di Fisica Nucleare - Laboratori Nazionali di Frascati, ITALY \\ "Istituto di Chimica dei Materiali, CNR-Area della Ricerca di Montelibretti - Roma, ITALY \\ ฯ Istituto di Struttura della Materia, CNR, sez. Trieste - Trieste, ITALY \\ ${ }^{\sharp}$ Istituto di Struttura della Materia, CNR-Area della Ricerca di Tor Vergata - Roma, ITALY
}

We have investigated the electronic and magnetic structures of $\mathrm{Sr}_{2} \mathrm{FeMoO}_{6}$ employing site-specific direct probes, namely x-ray absorption spectroscopy with linearly and circularly polarized photons. In contrast to some previous suggestions, the results clearly establish that $\mathrm{Fe}$ is in the formal trivalent state in this compound. With the help of circularly polarized light, it is unambiguously shown that the moment at the Mo sites is below the limit of detection $\left(<0.25 \mu_{B}\right)$, resolving a previous controversy. We also show that the decrease of the observed moment in magnetization measurements from the theoretically expected value is driven by the presence of mis-site disorder between Fe and Mo sites.

PACS number(s): 75.25.+z, 75.30.Vn, 75.20.Hr

The observation of colossal magnetoresistance (CMR) in the perovskite mixed valent manganites has led to a renewed interest in ferromagnetic oxides. It is believed that the double exchange mechanism in presence of strong electron-phonon couplings arising from Jahn-Teller distortions is responsible for the observed properties in the manganites [1]. Recently, double perovskite $\mathrm{Sr}_{2} \mathrm{FeMoO}_{6}$ was established as a new CMR material [2]. This compound, in contrast to the manganites, has certain technologically desirable properties, such as a substantial MR at a low applied field even at the room temperature. From a fundamental point of view, it is even more important to note that crystallographic data does not indicate any JT distortion and the lattice does not appear to play any significant role in this compound. Furthermore, the system is an undoped one in contrast to the manganites. Thus, $\mathrm{Sr}_{2} \mathrm{FeMoO}_{6}$ is in principle a simpler system to understand its properties in detailed theoretical terms. Inspite of this apparent simplicity, there are surprisingly many open issues concerning the basic electronic and magnetic structures of this compound.

In this compound, each of the $\mathrm{Fe}^{3+}(S=5 / 2)$ and $\mathrm{Mo}^{5+}(S=1 / 2)$ sublattices are believed to be arranged ferromagnetically, while the two sublattices are coupled to each other antiferromagnetically. It has been suggested 2 that the system is in a half metallic ferrimagnetic (HMFM) state leading to its fascinating CMR properties. However, there appears to be several controversies concerning the real nature of this compound. One neutron diffraction study [3] reported the absence of any moment at the Mo sites, suggesting Mo to be essentially non- magnetic, whereas another study [4] suggested $\sim 1 \mu_{B}$ at each Mo site. Moreover, analysis of Mössbauer results have been interpreted both in terms of $\mathrm{Fe}^{3+}$ [5, 6] and $\mathrm{Fe}^{2.5+}$ [7] states. Thus, it is obvious that even the basic issues concerning the electronic and magnetic structures of this compound have not been settled so far. Since the analysis of neutron and Mössbauer data are model dependent, it is obviously necessary to obtain site-specific direct information concerning the electronic and magnetic properties of this compound. Additionally, in the originally proposed magnetic structure [2], the system is expected to have a moment of $4 \mu_{B}$ per formula unit (f.u.) due to the ferrimagnetic coupling between the $\mathrm{Fe}^{3+} 3 d^{5}$ and $\mathrm{Mo}^{5+} 4 d^{1}$ configurations. However, the observed saturation magnetization $\left(M_{S}\right)$ is often found [2,8,9] to be about $3 \mu_{B} /$ f.u. The origin of this reduction in the magnetic moment is also not clear at present. We address all these issues combining linear and circularly polarized x-ray absorption spectroscopy (XAS), with its ability to provide direct, site-specific electronic and magnetic informations. In addition to providing the magnetic structure of this compound and explaining the reduction in the observed moment, our results also suggest that this compound cannot be described within the conventional double exchange mechanism.

$\mathrm{Sr}_{2} \mathrm{FeMoO}_{6}$ can exist with varying extent of mis-site disorder between the Fe and Mo sublattices. Synthesis and characterization of highly ordered $(\sim 90 \%)$ and extensively disordered (ordering of $\sim 30 \%$ ) $_{2} \mathrm{FeMoO}_{6}$ have been described in our earlier publication [6]. The experiments were carried out at the $4.2 \mathrm{R}$ circularly polarized beamline at Elettra Synchrotron Radiation Facility. The measurements were performed at $77 \mathrm{~K}$, which is well below the magnetic ordering temperature $(\sim 420 \mathrm{~K})[10]$. The sample surface was cleaned in situ by scraping with a diamond file. The degree of circular polarization at the relevant photon energy was approximately $85 \%$.

In order to address the valence state of $\mathrm{Fe}$ in such oxides, it is most suitable to probe the Fe $2 p_{3 / 2}\left(L_{3}\right)$ XAS which exhibits very clear differences between formal $\mathrm{Fe}^{2+}$ and $\mathrm{Fe}^{3+}$ states. Specifically, the $2 p_{3 / 2}$ absorption edge of all $\mathrm{Fe}^{2+}$ species in an octahedral crystal field exhibits a main peak at a lower energy, followed by a weaker peak or shoulder at a higher energy. The ordering of the peaks is reversed for $\mathrm{Fe}^{3+}$ species [11] providing an easy way to identify the formal $\mathrm{Fe}$ valence state independent of the 
extent of covalency. We have recorded a high resolution $(\sim 0.3 \mathrm{eV}) \mathrm{Fe} 2 p_{3 / 2}$ absorption spectrum of $\mathrm{Sr}_{2} \mathrm{FeMoO}_{6}$ with linearly polarized light (Fig. 1). From this figure, it is evident, even in absence of any detailed analysis that only a $\mathrm{Fe}^{3+}$ valence state is consistent with the experimental result, exhibiting a weaker lower energy shoulder and a higher energy main peak. However, in order to provide a quantitative description of the spectral features and, more importantly, of the ground state, it is important to carry out detailed calculations including hybridization effects with the ligands within a cluster model on an equal footing as core-valence and valence-valence multiplet interactions and crystal-field effects, as the participation of the ligand levels in determining the spectral features may be significant [12]. In order to minimize the number of free parameters, we fix the multiplet interaction strengths, $F_{d d}^{2}(9.7 \mathrm{eV}), F_{d d}^{4}(6.1 \mathrm{eV}), F_{p d}^{2}(5.4 \mathrm{eV})$, $G_{p d}^{1}(3.9 \mathrm{eV})$, and $G_{p d}^{3}(2.2 \mathrm{eV})$ to $80 \%$ of the atomic Hartree-Fock values to account for the solid state screening. Additionally, the hopping parameter strengths, $p d \sigma$, $p d \pi$, and $p p \sigma$ of $-1.7,0.9$ and $0.45 \mathrm{eV}$, respectively, are guided by a tight-binding fitting 13] of the spin-polarized $a b$ initio band dispersions. Moreover, we fix the multiplet averaged $2 p$ core - $3 d$ valence Coulomb interaction strength, $U_{p d}$, to be 1.2 times that of $U_{d d}$ between the $3 d$ electrons, according to the usual practice. Thus, we are left with only two adjustable parameters, namely $U_{d d}$ and the O $2 p-$ Fe $3 d$ charge transfer energy, $\Delta$. Since the resulting many-body problem within a complete basis approach 12] involves nearly 30,000 basis states, the calculations were carried out within the Lanczos algorithm. We obtain a remarkably good description of the spectral features with $U_{d d}=4 \mathrm{eV}$ and $\Delta=3 \mathrm{eV}$ for the $\mathrm{Fe}^{3+}$ configuration, as shown in Fig. 1. $U_{d d}$ and $\Delta$ estimated here are consistent with those in other octahedral $\mathrm{Fe}^{3+}$ oxides. The many-body ground state has $60.2 \% d^{5}$, $34.5 \% d^{6} \underline{L}^{1}$ and $5.1 \% d^{7} \underline{L}^{2}$ character, suggesting the system to be somewhat more ionic than even $\mathrm{LaFeO}_{3}$ [14]. It should be noted here that it was not possible to describe the spectral features at all starting with a formal $\mathrm{Fe}^{2+}$ configuration and then including configuration interaction for any choice of parameter strengths, conclusively establishing the $3+$ valence state of $\mathrm{Fe}$ in $\mathrm{Sr}_{2} \mathrm{FeMoO}_{6}$.

While the XAS with linearly polarized light at the Fe $2 p$ edge provides a definitive description of the sitespecific electronic structure, it is not as specific to the magnetic structure as x-ray magnetic circular dichroism (XMCD) results would be. In order to specifically investigate the magnetic structure, we have carried out XAS at the Fe $2 p$ and Mo $3 p$ edges with circularly polarized light. We use a lower resolution $(\sim 1.0 \mathrm{eV}$ at the Fe $2 p$ edge) to improve substantially the signal-to-noise ratio, though this smoothens out the detailed spectral features which are very similar to those with linearly polarized light (Fig. 1). In Fig. 2(a), we show the photon-flux normalized polarization-dependent Fe $2 p$ XAS spectra, $\mu^{+}$and $\mu^{-}$for highly ordered $\mathrm{Sr}_{2} \mathrm{FeMoO}_{6}$, corresponding to the helicity parallel and anti-parallel to the Fe $3 d$ majority-spin direction, respectively. The XMCD spectra, $\triangle \mu=\mu^{+}-\mu^{-}$, also shown in the same panel, clearly shows a substantial magnetic signal, indicative of a large Fe moment. The corresponding experimental $\mu^{+}, \mu^{-}$and $\triangle \mu$ spectra for Mo $3 p$ edge are shown in Fig. 2(b). It is evident from the XMCD spectrum of Mo that any magnetic moment at these sites is below the detection limit $\left(<0.25 \mu_{B}\right)$. The present result is in clear contradiction with the suggestion of a measurable moment $\left(\sim 1 \mu_{B}\right)$ at the Mo sites [2, 迎, while it is in agreement with a previous neutron diffraction measurement [3] where no moment could be detected at the Mo sites.

The above results, in conjunction with already known properties of $\mathrm{Sr}_{2} \mathrm{FeMoO}_{6}$, provide an understanding of the magnetic structure. The XMCD spectra establish a large moment at the Fe site, while negating the possibility of a substantial moment at the Mo sites. However, the electronic structure of this compound with a formal $\mathrm{Fe}^{3+}$ state requires the existence of another electron, nominally associated with a $\mathrm{Mo}^{5+} 4 d^{1}$ configuration and being responsible for the metallic behavior. Our results establish that the spin density arising from this single electron is not substantially at the Mo site. Since this electron is delocalized, it is not unreasonable to expect that the wavefunction of the electron will be spread over several sites. Band structure results, based on spin-polarized LMTO-GGA calculations [2]15] in fact clearly show that the states at the $E_{F}$ are almost equally contributed by $\mathrm{Fe} 3 d$, Mo $4 d$ and $\mathrm{O} 2 p$ states, suggesting an average of $\sim 0.3 \mu_{B}$ down-spin density at each Fe, Mo and six oxygen sites. The present experimental result at the Mo $3 p$ edge suggets that the spin-density is in reality further reduced $\left(<0.25 \mu_{B}\right)$ at the Mo sites, compared to the single particle calculations. Thus, it appears that the $\mathrm{FeO}_{6}$ octahedron carry more than $0.75 \mu_{B}$ down-spin density, rather than $\sim 0.6 \mu_{B}$ suggested by the band structure results. The suggestion of a substantial down-spin moment contribution at the Fe site is supported by our manybody cluster calculations (see Fig. 1), where the ground state wavefunction is found to have an average down-spin $d$ occupancy of 0.45 , somewhat larger than that suggested by the band structure results. Combining all these evidences, it would appear that the delocalized electron spin density is transferred from the minority spin of the Mosites via hybridization to $\mathrm{Fe}(\sim 45 \%)$ and $\mathrm{O}(\geq 30 \%)$ with less than about $25 \%$ of spin-density at the Mo site, thereby spreading over several sites. Thus, it appears that the delocalized spin density, antiferromagnetically coupled to the localized up spins at the Fe sites, prefers to be spatially closer to the central Fe sites, thereby gaining a stronger antiferromagnetic coupling [16] between the localized and the delocalized spins rather than residing at the farther Mo sites. While the double exchange 
mechanism, applicable to the manganites, has often been invoked to describe these ordered double perovskite systems, the present results clearly suggest a new physics for this class of compounds compared to manganites. In the DE mechanism, the localized spin at the Mn site and the delocalized electrons, largely residing at the same atomic site, are coupled ferromagnetically. In contrast, the present system is reminiscent of previously discussed Zhang-Rice singlet formation in the context of high $\mathrm{T}_{C}$ cuprates [17]. In that case the localized moment at the central $\mathrm{Cu}$ site is coupled antiferromagnetically with the doped delocalized spin-density spread over the central $\mathrm{Cu}$ and the nearest neighbor oxygen sites to form a singlet state. In the present case, the localized Fe $S=5 / 2$ state couples antiferromagnetically with the spin density of delocalized $S=1 / 2$ state to form a $S=2$ state.

Having established the basic magnetic structure of $\mathrm{Sr}_{2} \mathrm{FeMoO}_{6}$, we now address the issue of consistently observing a lower $M_{S}$ value for this compound than is expected on the basis of the simple ionic picture, in all reported results. In this context, it is important to note that $\mathrm{Sr}_{2} \mathrm{FeMoO}_{6}$ always appears with a finite concentration of mis-site disorder where a pair of $\mathrm{Fe}$ and Mo exchange their crystallographic positions. The best compounds have been reported to have $\sim 90 \%$ ordering of the $\mathrm{Fe}$ and the Mo sites [2,6]. In order to investigate whether such a mis-site disorder can be responsible for the observed reduction of the moment from the ideal value of 4 to about $3 \mu_{B}$ and whether the reduction in the total magnetization is related to a corresponding loss in the local magnetic moment of $\mathrm{Fe}$, we have also recorded the XAS at the Fe $2 p$ edge of the extensively disordered $\mathrm{Sr}_{2} \mathrm{FeMoO}_{6}$ with circularly polarized light. These edges along with the XMCD result at Fe $2 p$ edges for the disordered sample are shown in Fig. 3(a), while in Fig. 3(b), we compare the XMCD signals from the ordered and disordered $\mathrm{Sr}_{2} \mathrm{FeMoO}_{6}$. It is evident from the spectra that the magnetic moment on individual Fe ions decreases remarkably with decreasing ordering. In order to quantify our results, we have calculated the orbital, spin and total moments at the Fe sites from these spectra with the assumption of negligible magnetic-dipole moment, using the well established sum rules [18, 19. All the individual spin, orbital and total moments of these two samples are shown in Table 1. In these cases, we find that $m_{\text {orb }}$ is very small, due to the approximately $3 d^{5}$ configuration of Fe ions. The resulting total moments estimated from the XMCD signals are 1.68 and $1.36 \mu_{B} / \mathrm{Fe}$ for the ordered and the disordered systems, respectively. It is to be noted that the magnetic moments obtained from the XMCD results are considerably smaller than the total magnetic moments obtained from bulk magnetization measurements [2,96. Such discrepancies are well known in the literature [20], and may arise from many factors. It has been variously attributed to uncertainties in data analysis, limitations of the applicability of the atomic sum rules arising from non-ideal geometry in real experiments and/or solid-state effects, and non-saturation of the magnetization at modest magnetic fields near the surface region. Thus, the absolute value of the moment estimated from the XMCD results is per se not a useful quantity, though the extensive XMCD literature shows that relative changes in the magnetic moments estimated from XMCD is a very reliable quantity. For our purpose, we first establish this point explicitly by comparing the XMCD results (Fig. 3 inset) of the ordered $\mathrm{Sr}_{2} \mathrm{FeMoO}_{6}$ with a bulk magnetic moment of $\sim 2.81 \mu_{B} /$ f.u. at $77 \mathrm{~K}$ and a closely related compound, $\mathrm{Sr}_{2} \mathrm{FeMo}_{0.3} \mathrm{~W}_{0.7} \mathrm{O}_{6}$, with a bulk magnetic moment of $3.64 \mu_{B} /$ f.u. at $77 \mathrm{~K}$. The XMCD results (Table 1) measured at the same temperature clearly suggest an approximately $42 \pm 2 \%$ drop in the XMCD moment compared to the bulk one for both the samples. Thus, having established the efficacy of probing the relative changes of magnetization in these and related systems employing XMCD, our results on the ordered and disordered $\mathrm{Sr}_{2} \mathrm{FeMoO}_{6}$, shown in Fig. 3 and Table 1, clearly establish a remarkable decrease in the magnetic moment at the Fe sites with increasing mis-site disorder. These experimental results are also consistent with the recent band structure calculations [15] within a supercell for mis-site disorders between the $\mathrm{Fe}$ and Mo occupancies. The band structure results suggest that a complete disorder would result in a $34 \%$ decrease in the moment at the Fe sites, while a $50 \%$ order would have a $21 \%$ decrease of the moment compared to the fully ordered sample. The present experimental result of a $23 \%$ decrease in the Fe moment for the "disordered" sample with about $30 \%$ ordering is consistent with these band structure results. Thus, it appears that the decrease in the magnetic moment invariably observed for the so-called ordered $\mathrm{Sr}_{2} \mathrm{FeMoO}_{6}$ is mainly due to the presence of finite $(\sim 10 \%)$ mis-site disorder. The origin of the decrease in the $\mathrm{Fe}$ moment in presence of mis-site disorder is essentially due to the destruction of the half-metallic ferromagnetic state of the fully-ordered ideal system, thereby transferring $d$-electrons from the up-spin to the downspin bands 15 .

In summary, site-specific x-ray absorption spectroscopy with linearly polarized light established the formal valency of $\mathrm{Fe}$ in $\mathrm{Sr}_{2} \mathrm{FeMoO}_{6}$ to be $3+$. Detailed investigation of $\mathrm{x}$-ray magnetic circular dichroism data confirms a large moment at the Fe site. Our results provide a direct evidence for a negligible $\left(<0.25 \mu_{B}\right)$ magnetic moment at the Mo site, thereby suggesting that the delocalized electron spin density, coupled antiferromagnetically to the localized Fe-spins, is delocalized over several sites including the neighboring $\mathrm{FeO}_{6}$ octahedra and indicating a novel origin of magnetism, different from the conventional double exchange mechanism. A comparison of XMCD results from the ordered and the disordered samples establishes that the presence of mis-site disorder 
between the Fe and Mo sites even in the so-called ordered samples is responsible for the observed drop in the magnetic moment from the expected value of $4 \mu_{B} /$ f.u. to experimentally observed value of about $3 \mu_{B} /$ f.u.

This project is supported by DST, Government of India and Italian Ministry of Science under the program of cooperation in Science and Technology. We thank C. Carbone and P. Mahadevan for useful discussions.

$\infty \quad$ Also at Jawaharlal Nehru Centre for Advanced Scientific Research, Bangalore, India. sarma@sscu.iisc.ernet.in

[1] A.J. Millis, P.B. Littlewood, and B.I. Shraiman, Phys. Rev. Lett. 74, 5144 (1995); A.J. Millis, B.I. Shraiman, and R. Mueller, Phys. Rev. Lett. 77, 175 (1996).

[2] K. -I. Kobayashi et al., Nature 395, 677 (1998).

[3] B. Garc'ia-Landa et al., Solid State Commun. 110, 435 (1999).

[4] Y. Moritomo et al., J. Phys. Soc. Jpn. 69, 1723 (2000).

[5] T. Nakagawa, K. Yoshikawa and S. Nomura, J. Phys. Soc. Jpn. 27, 880 (1969).

[6] D. D. Sarma et al., Solid State Commun. 114, 465 (2000).

[7] J. Lindén et al., Appl. Phys. Lett. 76, 2925 (2000).

[8] K. -I. Kobayashi et al., J. Magn. Mag. Mater. 218, 17 (2000).

[9] S. Ray et al., J. Phys.: Condens. Matter 13, 607 (2001).

[10] T. Nakagawa, J. Phys. Soc. Jpn. 24, 806 (1968).

[11] G. van der Laan and I.W. Kirkman, J. Phys : Condens. Matter 4, 4189 (1992).

[12] P. Mahadevan and D. D. Sarma, Phys. Rev. B 61, 7402 (2000).

[13] P. Mahadevan and T. Saha-Dasgupta, unpublished results.

[14] A. Chainani, M. Mathew and D.D. Sarma, Phys. Rev. B 48, 14818 (1993).

[15] T. Saha-Dasgupta and D.D. Sarma, Phys. Rev. B (to appear).

[16] D.D. Sarma et al., Phys. Rev. Lett. 85, 2549 (2000); D.D. Sarma, Current Op. Solid St. Mat. Sci. 5(4), to appear in 2001.

[17] F.C. Zhang and T.M. Rice, Phys. Rev. B 37, 3759 (1988)

[18] B. T. Thole et al., Phys. Rev. Lett. 68, 1943 (1992); P. Carra et al., Phys. Rev. Lett. 70, 694 (1993).

[19] Y. Teramura, A. Tanaka and T. Jo, J. Phys. Soc. Jpn. 65, 1053 (1996).

[20] J.-H. Park et al., Phys. Rev. Lett. 81, 1953 (1998); J. Okamoto et al., Phys. Rev. B 62, 4455 (2000).

Table 1. Spin moments, Orbital moments and Total moments in $\mu_{B} / \mathrm{Fe}$ at $77 \mathrm{~K}$.

\begin{tabular}{cccc}
\hline \hline Compound & $M_{\text {spin }}$ & $M_{\text {orb }}$ & $M_{\text {tot }}$ \\
\hline Ordered $\mathrm{Sr}_{2} \mathrm{FeMoO}_{6}$ & 1.71 & $-3.6 \times 10^{-2}$ & 1.68 \\
Disordered $\mathrm{Sr}_{2} \mathrm{FeMoO}_{6}$ & 1.44 & $-7.6 \times 10^{-2}$ & 1.36
\end{tabular}

\begin{tabular}{llll}
$\mathrm{Sr}_{2} \mathrm{FeMo}_{0.3} \mathrm{~W}_{0.7} \mathrm{O}_{6}$ & 2.15 & $-7.3 \times 10^{-2}$ & 2.07 \\
\hline \hline
\end{tabular}

FIG. 1. Experimental and calculated Fe $2 p_{3 / 2}$ x-ray absorption spectrum for ordered $\mathrm{Sr}_{2} \mathrm{FeMoO}_{6}$.

FIG. 2. x-ray absorption spectra at (a) Fe $2 p$, and (b) Mo $3 p$ edges for ordered $\mathrm{Sr}_{2} \mathrm{FeMoO}_{6}$, measured using circularly polarized light. In the lower panels, circular dichroism signals, the difference between the absorption for right and left circularly polarized light at these edges are shown. The integral difference spectrum for $\mathrm{Fe} 2 p$ edge is also shown in the lower panel of (a).

FIG. 3. (a) x-ray absorption spectra at Fe $2 p$-edge for disordered $\mathrm{Sr}_{2} \mathrm{FeMoO}_{6}$, measured using circularly polarized light and the corresponding XMCD signal. (b) XMCD signals for the ordered and disordered $\mathrm{Sr}_{2} \mathrm{FeMoO}_{6}$. In the inset, the XMCD signals for ordered $\mathrm{Sr}_{2} \mathrm{FeMoO}_{6}$ and $\mathrm{Sr}_{2} \mathrm{FeMo}_{0.3} \mathrm{~W}_{0.7} \mathrm{O}_{6}$ are shown. 


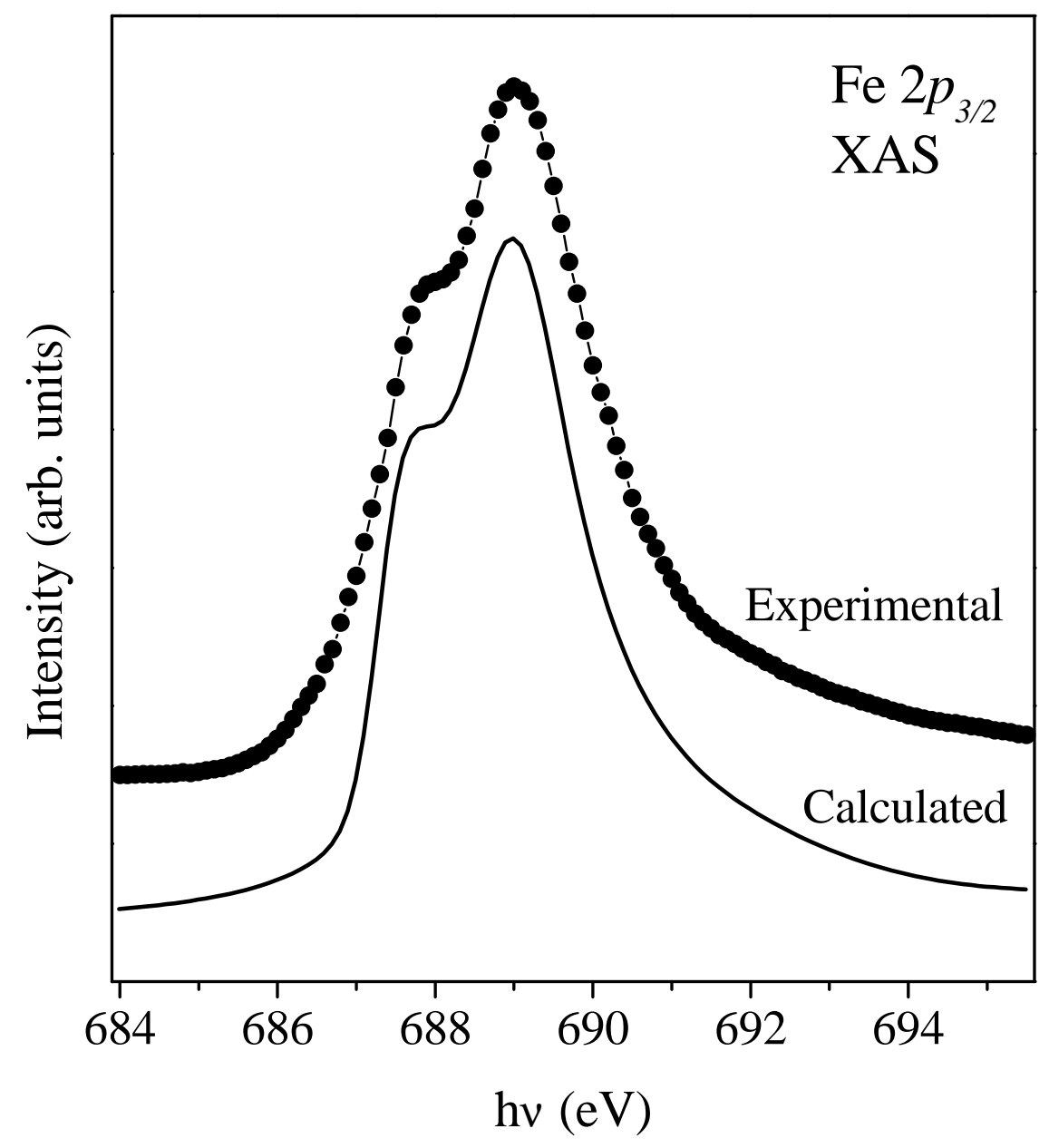

Fig. 1 

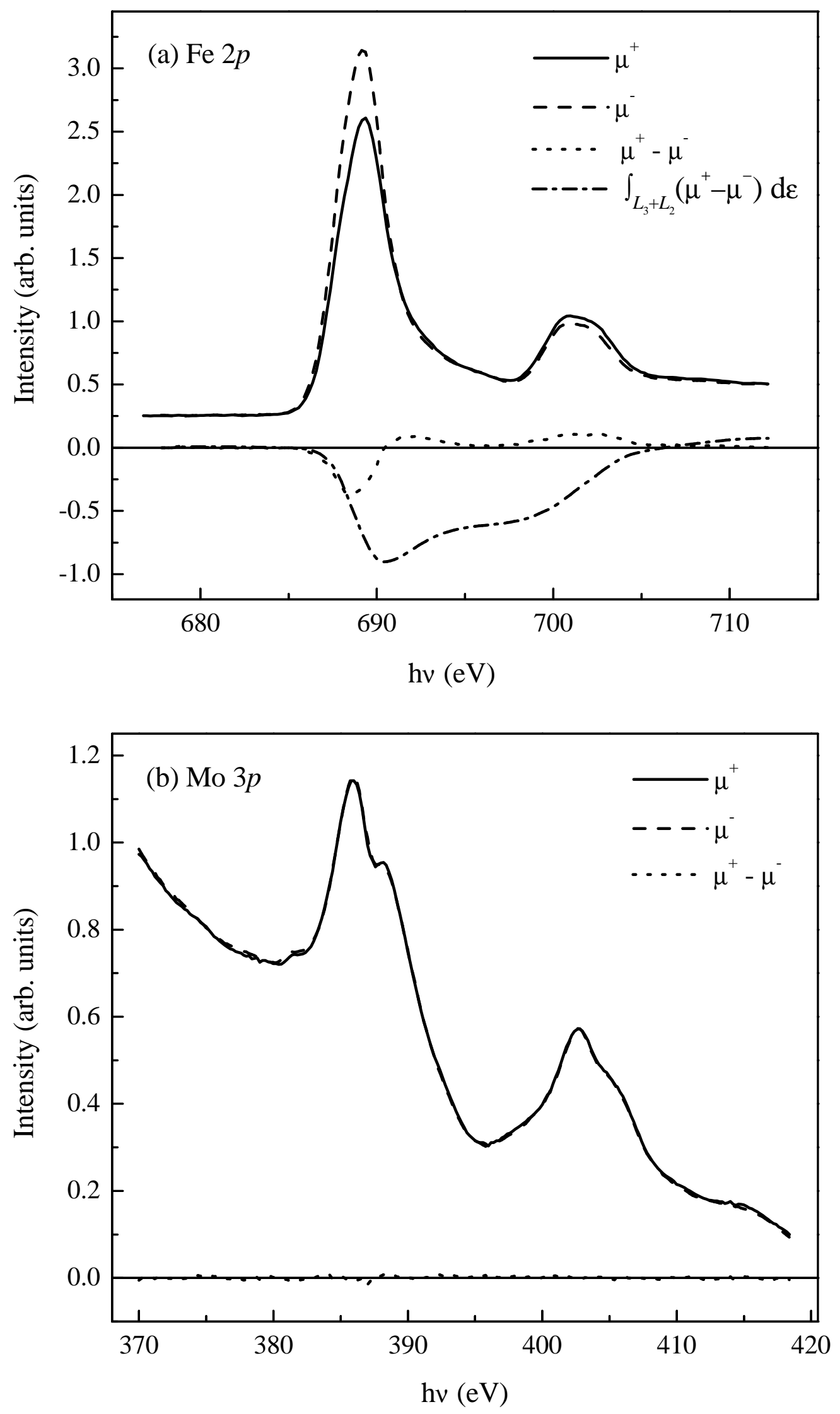

Fig. 2 

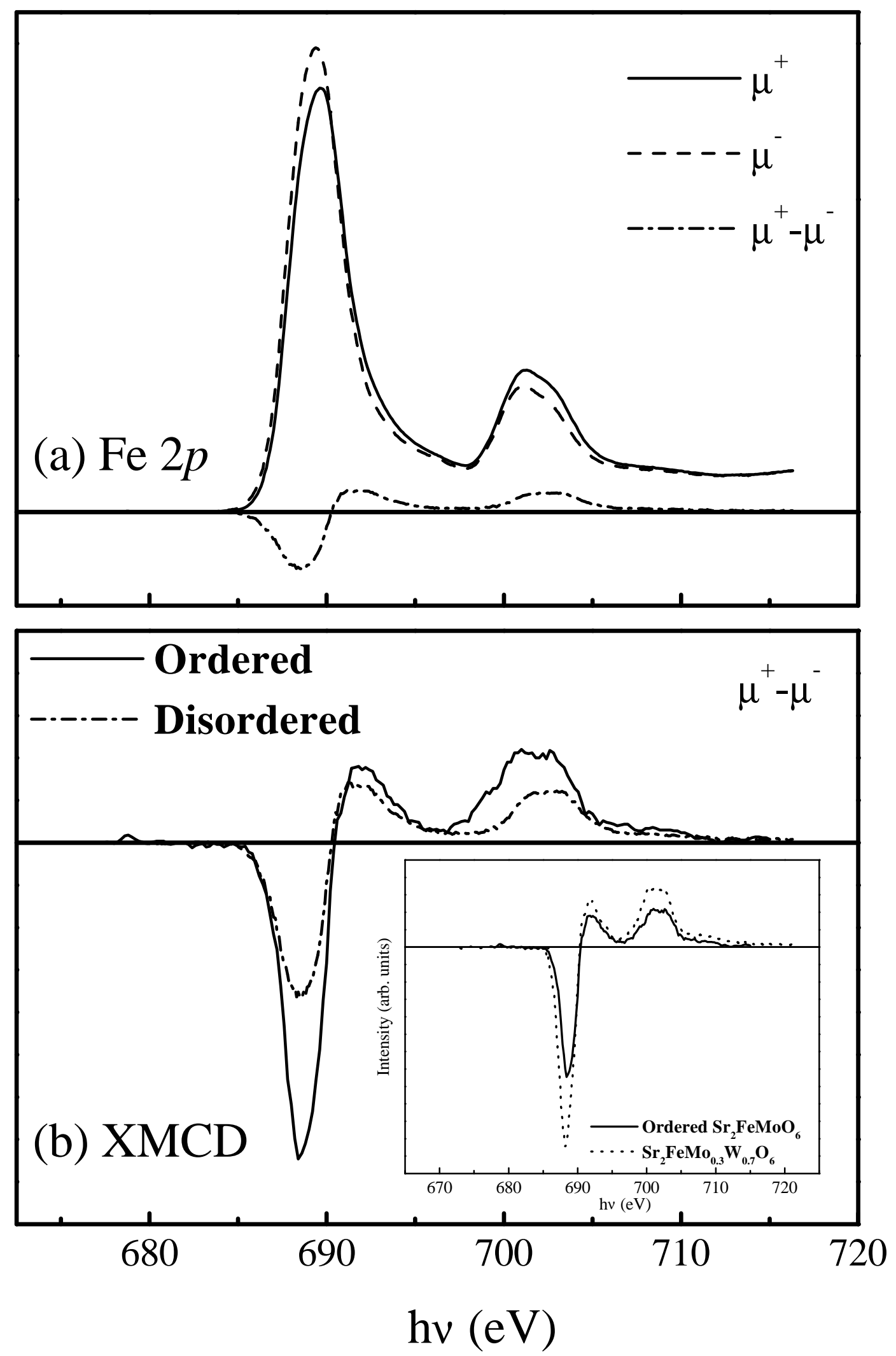

Fig. 3 